\title{
Optimal panel of immunohistochemistry for the diagnosis of B-cell non-Hodgkin lymphoma using bone marrow biopsy: a tertiary care center study
}

\author{
Nisha Marwah ${ }^{1}$, Manali Satiza ${ }^{1}$, Niti Dalal ${ }^{1}$, Sudhir Atri ${ }^{2}$, Monika Gupta $^{1}$, Sunita Singh ${ }^{1}$, Rajeev Sen ${ }^{1}$ \\ Departments of ${ }^{1}$ Pathology and ${ }^{2}$ Medicine, Pt. B. D. Sharma PGIMS, Rohtak, India
}

\section{p-ISSN 2287-979X / e-ISSN 2288-0011 https://doi.org/10.5045/br.2021.2020146 Blood Res 2021;56:26-30.}

Received on June 22, 2020

Revised on December 17, 2020

Accepted on December 17, 2020

\author{
Correspondence to \\ Niti Dalal, M.D. \\ Department of Pathology, Pt. B. D. Sharma, \\ PGIMS, Rohtak, Haryana 124001, India \\ E-mail: drnitidalal@gmail.com \\ (C) 2021 Korean Society of Hematology
}

\section{Background}

Morphological diagnosis of non-Hodgkin lymphoma (NHL) is usually based on lymph node biopsy. Bone marrow biopsy (BMB) is important for staging, and morphology alone can be challenging for subtyping. Immunohistochemistry (IHC) allows a more precise diagnosis and characterization of NHL using monoclonal antibodies. However, there is a need for a minimal panel that can provide maximum information at an affordable cost.

\section{Methods}

All newly diagnosed cases of B-cell NHL with bone marrow infiltration between 2017 and 2019 were included. BMB was the primary procedure for diagnosing B-cell NHL. Subtyping of lymphomas was performed by immunophenotyping using a panel of monoclonal antibodies on IHC. The primary diagnostic panel of antibodies for B-cell NHL included CD19, CD20, CD79, CD5, CD23, CD10, Kappa, and Lambda. The extended panel of antibodies for further subtyping included CD30, CD45, CD56, Cyclin D1, BCL2, and BCL6.

\section{Results}

All cases of B-cell NHL were classified into the chronic lymphocytic leukemia (CLL) and non-CLL groups based on morphology and primary IHC panel. In the CLL group, the most significant findings were CD5 expression, CD23 expression, dim CD79 expression, and weak surface immunoglobulin (Ig) positivity. In the non-CLL group, they were CD5 expression, positive or negative CD23 expression, strong CD79 expression, and strong surface Ig expression. An extended panel was used for further subtyping of non-CLL cases, which comprised CD10, Cyclin D1, BCL2, and BCL6.

\section{Conclusion}

We propose a two-tier approach for immunophenotypic analysis of newly diagnosed B-cell NHL cases with a minimum primary panel including CD5, CD23, CD79, Kappa, and Lambda for differentiation into CLL/non-CLL group and Kappa and Lambda for clonality assessment. An extended panel may be used wherever required for further subtyping of non-CLL.

Key Words Non-Hodgkin lymphoma, Immunohistochemistry, Chronic lymphocytic leukemia, Non-chronic lymphocytic leukemia, Bone marrow biopsy

\section{INTRODUCTION}

Non-Hodgkin lymphoma (NHL) is a heterogeneous group of chronic lymphoproliferative disorders originating in the $\mathrm{B}$, $\mathrm{T}$, or natural killer (NK) lymphocytes. It is characterized by abnormal proliferation of monoclonal lymphocytes in the peripheral blood, the bone marrow, the lymph node, or any other lymphoid tissue and is a major health concern globally. B-cell lymphoma represent $80-85 \%$ of all NHL cases, while T-cell lymphoma represent $15-20 \%$ of all NHL cases; NK lymphoma is rare [1].

Morphological diagnosis of NHL is based on lymph node biopsy. In patients with NHL, bone marrow biopsy (BMB) 
is an important for staging. However, the morphological evaluation by $\mathrm{BMB}$ to assess the involvement in the lymphoid malignancies can be challenging [2]. A comprehensive diagnosis of chronic lymphocytic leukemia (CLL) is essential to differentiate it from other NHL groups and for risk stratification, which is crucial for deciding the therapeutic approach [3].

Immunohistochemistry (IHC) allows a more precise diagnosis and characterization of NHL. IHC is useful for distinguishing between reactive and neoplastic lymphoid infiltration [4-6]. IHC also provides a visual impression of the patterns of infiltration and architectural relationship in addition to allowing long-term preservation and providing the feasibility of re-examination at any time [7].

The unique feature of lymphoma is clonal proliferation of lymphocytes arrested at different stages of differentiation, which recapitulates the stages of normal lymphocyte differentiation. IHC with various antibodies identifies the specific lineage and developmental stage of lymphoma [8].

Given the importance of cost optimization in the Indian healthcare system, an appropriate panel that would provide an accurate diagnosis at an affordable cost is crucial. Various studies have been conducted to investigate the utility of different markers and their combinations in the diagnosis and sub-typing of various types of NHL. The economic challenges in India occasionally make cost management more difficult, which has created the need to develop basic minimal screening panels. Hence, this study aimed to develop a primary screening panel that can differentiate the CLL group of NHL from the non-CLL group of NHL and a secondary panel for further subtyping of the non-CLL group wherever necessary.

\section{MATERIALS AND METHODS}

The study included a total of 30 newly diagnosed cases of B-cell NHL with bone marrow infiltration over a period of 2 years from 2017 to 2019. All patients were included in the study after obtaining ethical clearance from the institutional ethical committee. All previously treated cases, those with precursor lymphoblastic lymphoma/leukemia, those with inadequate length of bone marrow biopsy samples $(<1.5 \mathrm{~cm})$, and those that did not show infiltration on bone marrow biopsy were excluded from the study.

$\mathrm{BMB}$ was the primary procedure for diagnosing B-cell NHL. All specimens were processed using routine histological techniques for paraffin embedding, and morphological assessment was performed using routine hematoxylin and eosin staining. Subtyping of the lymphoma was performed by immunophenotyping (IPT) using a panel of monoclonal antibodies on IHC (Table 1). The primary diagnostic panel of antibodies for B-cell NHL included CD19, CD20, CD79, CD5, CD23, CD10, Kappa, and Lambda. The extended panel of antibodies for further subtyping included CD30, CD45, CD56, Cyclin D1, BCL2, and BCL6.

The immunophenotypic diagnostic criteria depending on the expression of antibodies were used for subtyping of chronic lymphoproliferative disorders, as shown in Table 1 [9].

\section{Statistics}

SPSS 20.0 (IBM, Armonk, NY, USA) software was used for the statistical analysis. Kappa and lambda tests were also performed. Differences between the groups were considered significant only when the $P$-value was $<0.05$.

\section{RESULTS}

The mean patient age was 56.4 years (range, 21-86 yr). On complete blood examination, most cases (66.6\%) had an elevated absolute lymphocyte count and atypical lymphocytosis on peripheral blood film. The most common clinical presentation was lymphadenopathy along with hepatomegaly or splenomegaly (63.3\% cases). All cases were further sub-

Table 2. Distribution of cases on the basis of bone marrow aspiration findings.

\begin{tabular}{lcc}
\hline \multicolumn{1}{c}{ BMA findings } & N of cases & $\%$ \\
\hline Chronic lymphoproliferative disorder & 19 & 63.3 \\
Chronic lymphocytic leukemia & 4 & 13.3 \\
Atypical lymphocytosis & 5 & 16.6 \\
Dry tap aspirate & 2 & 6.66 \\
Total cases & 30 & 100 \\
\hline
\end{tabular}

Abbreviation: BMA, bone marrow aspiration.

\begin{tabular}{|c|c|c|c|c|c|c|c|c|c|}
\hline & CD19 & CD20 & $\mathrm{CD} 23$ & CD5 & CD79 & CD10 & BCL2 & BCL6 & Cyclin D1 \\
\hline CLL & + & + & + & + & $\operatorname{Dim}+$ & - & - & - & - \\
\hline NHL (unclassified) & + & + & + & + & + & - & - & - & - \\
\hline MCL & + & + & - & + & + & - & - & - & + \\
\hline $\mathrm{FL}$ & + & + & - & - & + & + & + & - & - \\
\hline DLBCL & + & + & - & + & + & + & - & + & - \\
\hline
\end{tabular}

Abbreviations: CLL, chronic lymphocytic leukemia; DLBCL, diffuse large B-cell lymphoma; $\mathrm{FL}$, follicular lymphoma; $\mathrm{MCL}$, mantle cell lymphoma; NHL, non-Hodgkin lymphoma. 

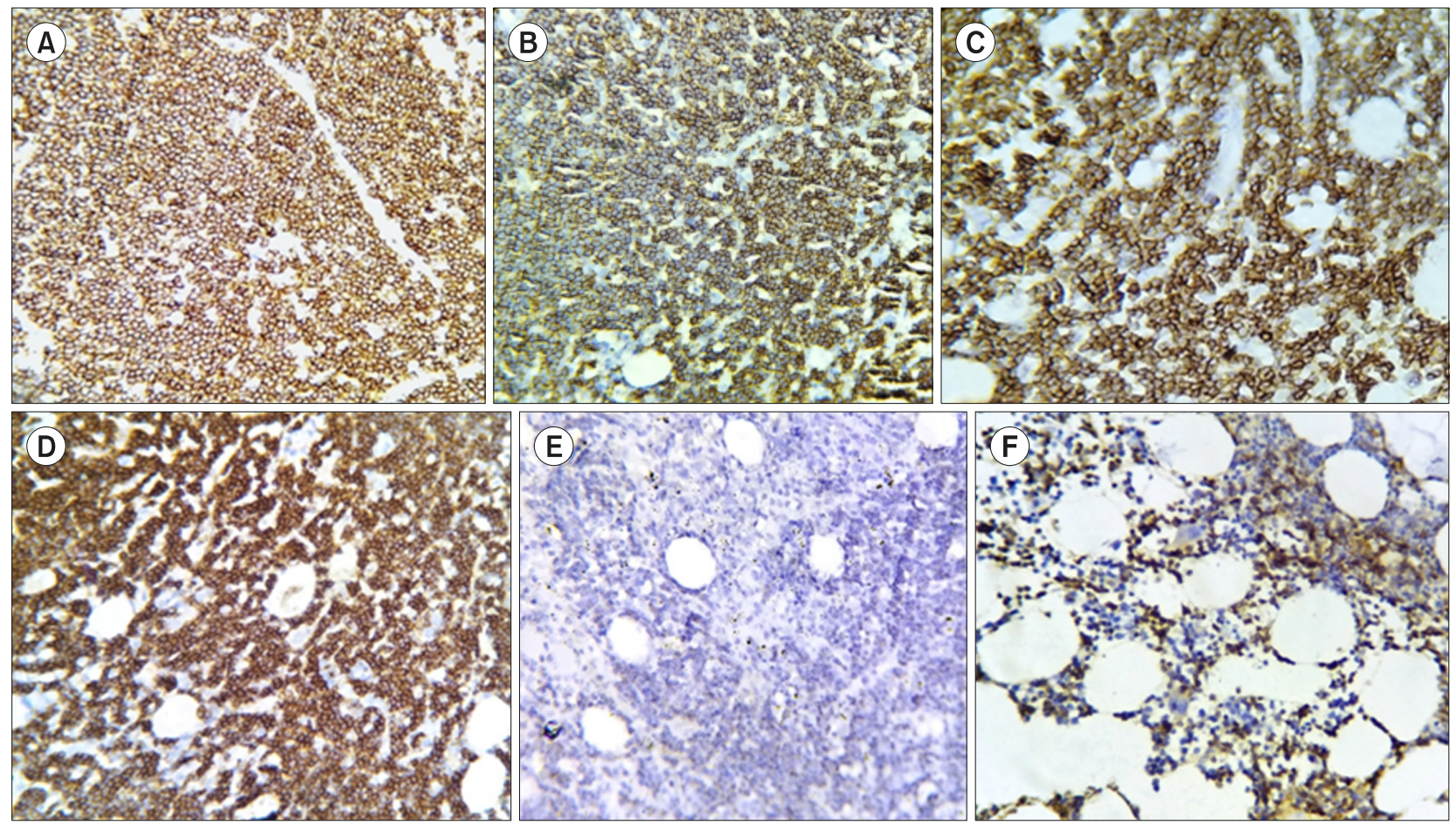

Fig. 1. Immunohistochemical profile of chronic lymphocytic leukemia showing CD19+, CD20+, CD5+, CD23+, CD79-, non-specific Lambda (immunohistochemistry, $\times 400$ ).

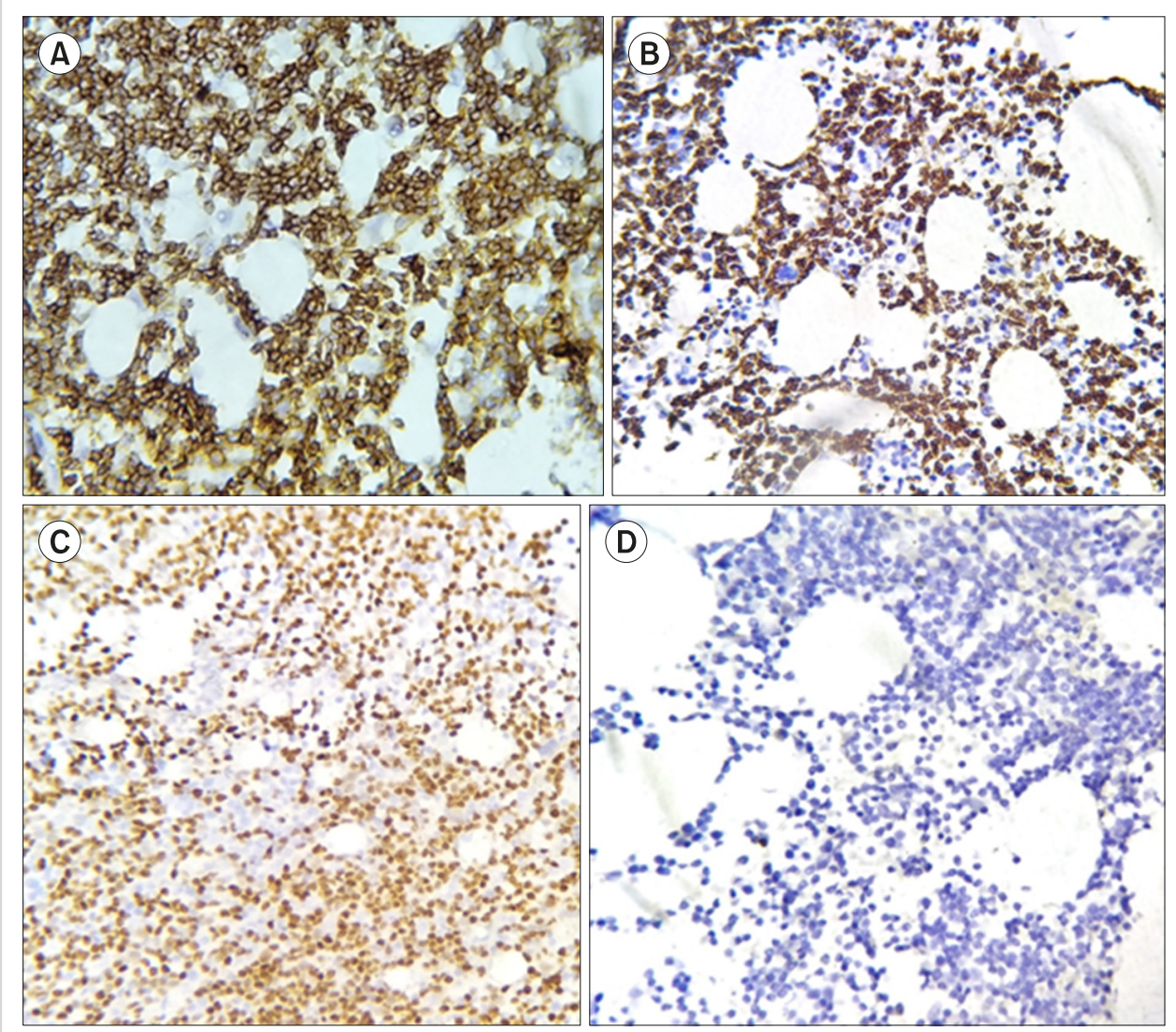

Fig. 2. Immunohistochemical profile of mantle cell lymphoma showing CD5+, CD79+, Cyclin D1+, CD23(immunohistochemistry, $\times 400$ ). 
jected to BMB and IPT by IHC. Varied patterns of bone marrow infiltration were observed on bone marrow biopsy, including diffuse (most common), nodular, interstitial, and mixed. Cases were initially classified into the CLL and non-CLL groups. Cases in the non-CLL group were further subtyped.

Bone marrow aspiration revealed that a chronic lymphoproliferative disorder was present in most cases (63.3\%). All cases were also subjected to BMB and IPT by IHC (Table 2).

Classification of cases: Of the 30 cases included, 12 were classified into the CLL group (Fig. 1) and 18 were classified into the non-CLL group. An extended panel comprising Cyclin D1, BCL2, and BCL6 was used for subtyping the non-CLL cases. According to IHC, the non-CLL group ( $\mathrm{N}=18)$ comprised four cases of mantle cell lymphoma (MCL) (Fig. 2), three cases of follicular lymphoma (FL) (Fig. 3), and two cases of diffuse large B-cell lymphoma (DLBCL). Nine of 18 cases were not subtyped and were considered B-cell NHL cases. Regarding light chain restriction, many cases were non-specific for Kappa (13/30) and Lambda (15/30) on IHC (Table 3).

\section{DISCUSSION}

Recent pathology relies on IPT for the accurate classification of lymphomas. The current World Health Organization classification suggests the classification of lymphomas should be based on morphology supplemented by IPT. In the current study, we have discussed the role of
IHC in the diagnosis and classification of NHL using BMB. A common primary panel, including CD19, CD20, CD5, CD10, CD23, CD79, CD45, kappa, and lambda, was used. CD5 expression, CD23 expression, dim CD79 expression, and weak surface immunoglobulin (Ig) positivity were the most significant findings in the CLL group. CD5 expression, positive or negative CD23 expression, strong CD79 expression, and strong surface Ig expression were the most common findings in the non-CLL groups. In our study, a screening panel comprising CD5, CD23, CD79, sIg, Kappa, and Lambda was adequate to distinguish between CLL and non-CLL groups. These findings were comparable to those

Table 3. Distribution of cases based on bone marrow biopsy and immunohistochemistry findings.

\begin{tabular}{lcc}
\hline \multicolumn{1}{c}{ IHC diagnosis } & N of cases & $\%$ \\
\hline CLL & 12 & 40 \\
CLL & 12 & 40 \\
Non-CLL & 18 & 60 \\
NHL (unclassified) & 9 & 30 \\
Mantle cell lymphoma & 4 & 13.3 \\
Marginal zone lymphoma & 0 & 0 \\
Hairy cell leukemia & 0 & 0 \\
Follicular lymphoma & 3 & 10 \\
Diffuse large B cell lymphoma & 2 & 6.66 \\
Total cases & 30 & 100 \\
\hline
\end{tabular}

Abbreviations: $\mathrm{CLL}$, chronic lymphocytic leukemia; IHC, immunohistochemistry; NHL, non-Hodgkin lymphoma.

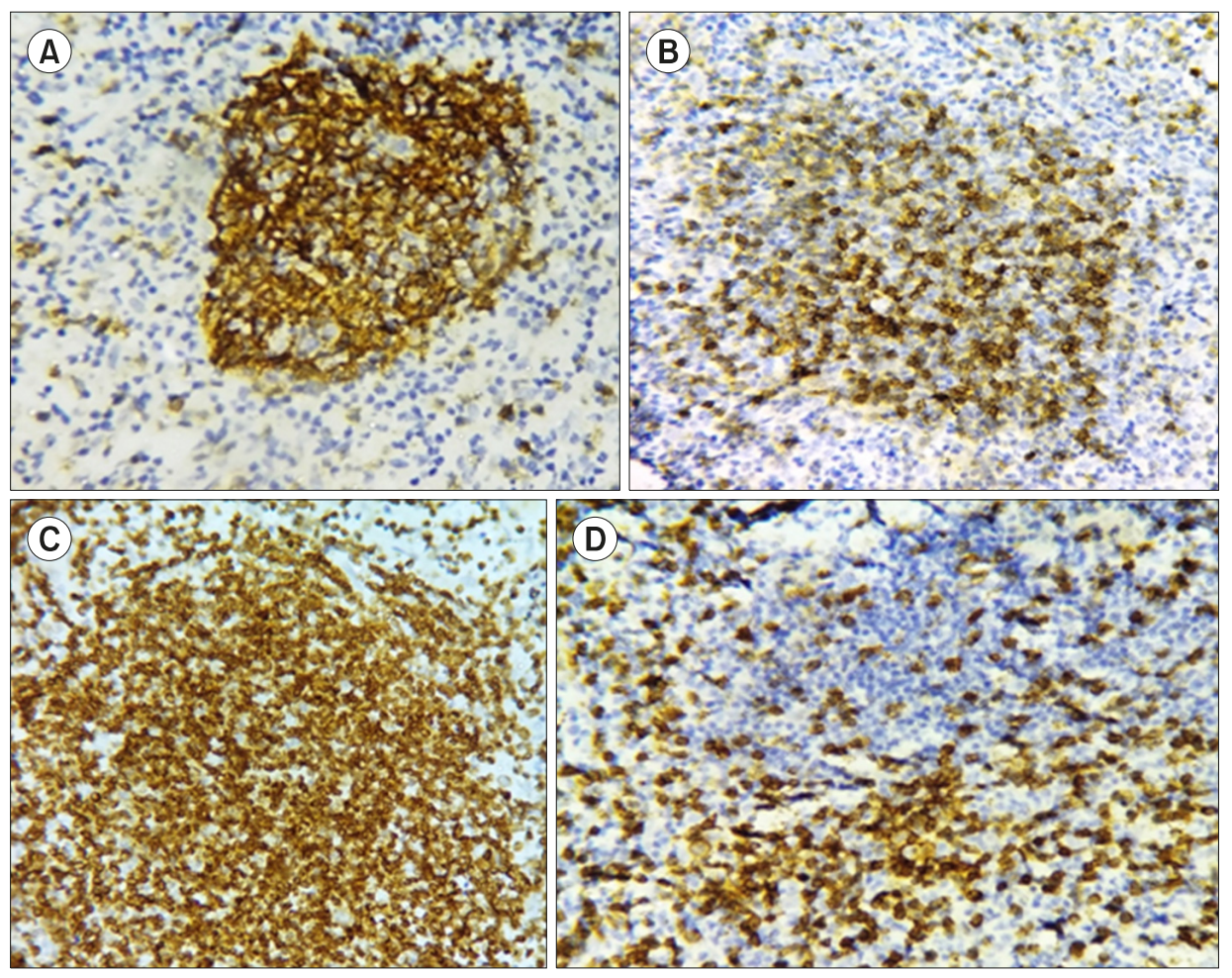

Fig. 3. Immunohistochemical profile of follicular lymphoma showing CD19+ in the lymphoid follicle, $\mathrm{CD} 10+$ in the lymphoid follicle, $\mathrm{BCL} 2+$ in the lymphoid follicle, CD5- B cells in the lymphoid follicle with CD5+ scattered T-cells in the background (immunohistochemistry, $\times 400$ ). 
reported by Dewan et al. [9], who divided CLL and non-CLL groups on the basis of positive reactivity to CD5 and CD23, weak SIg reactivity, and non-reactivity to FMC7 and CD79 [9]. An extended panel comprising Cyclin D1, BCL2, and BCL6 was used for subtyping of non-CLL cases.

Regarding light chain restriction, it was difficult to interpret the Ig light chain detection on IHC because of the background staining in paraffin-embedded blocks and antigen destruction by fixation and decalcification of $\mathrm{BMB}$ specimens. Many cases were non-specific for Kappa (13/30) and Lambda (15/30) on IHC. Similar findings were observed by Leers et al. [10], who reported the lack of contrast between surface Ig staining and extracellular Ig staining as a major drawback in the detection of monoclonality by IHC, and Abdel-Ghafar et al. [11], who observed destruction of some of the antigenic epitopes due to the fixation and decalcification processes. Based on our results, we propose a basic approach in the immunophenotypic analysis of all newly diagnosed cases of B-cell NHL. The screening panel should comprise the following markers: CD19, CD20 (B cell), CD23, CD79, CD5, CD10 (for further categorization), and Kappa and Lambda (monoclonality assessment). A limited primary panel comprising CD5, CD23, CD79, and sIg can be used to differentiate between CLL and non-CLL. The diagnosis of CLL, MCL, and marginal zone lymphoma (MZL) could be made in our study using the screening panel. Diagnosis of other types of B-cell NHL, including atypical CLL, hairy cell leukemia (HCL), FL, and DLBCL and confirmation of MCL in few cases require an extended panel comprising CD200, FMC7 (atypical CLL), CD25, CD103, CD123 (HCL), Cyclin D1 (MCL), BCL2 (FL), and BCL6 (DLBCL) on either flow cytometry or IHC. In most published articles, both IHC and flow cytometry have been reported to enhance the diagnostic potential of B-cell NHL and help the treating physician in planning the treatment accordingly.

Due to the unavailability of markers such as CD200, FMC7, CD25, CD123, and CD103 on IHC, the diagnosis of other types of NHL such as MZL and HCL was not possible. Another limitation of IHC in our study was non-specific and background staining for surface Igs (Kappa and Lambda).

A two-tier approach for the IHC panel can be adopted with a minimum primary panel for clonality assessment and differentiation into the CLL and non-CLL groups. An extended panel can be used whenever necessary for further subtyping and stratification.

The optimal minimal primary panel of antibodies for the diagnosis of B-cell NHL should be based on morphology. Depending on the findings of the primary panel, a secondary panel for further subtyping and stratification can be used.

\section{Authors' Disclosures of Potential Conflicts of Interest}

No potential conflicts of interest relevant to this article were reported.

\section{REFERENCES}

1. Zelenetz AD, Abramson JS, Advani RH, et al. NCCN Clinical Practice Guidelines in Oncology: non-Hodgkin's lymphomas. J Natl Compr Canc Netw 2010;8:288-334.

2. Sah SP, Matutes E, Wotherspoon AC, Morilla R, Catovsky D. A comparison of flow cytometry, bone marrow biopsy, and bone marrow aspirates in the detection of lymphoid infiltration in B cell disorders. J Clin Pathol 2003;56:129-32.

3. Okaly GV, Nargund AR, E V, Jayanna PK, Juvva CR, Prabhudesai S. Chronic lymphoproliferative disorders at an Indian tertiary cancer centre - the panel sufficiency in the diagnosis of chronic lymphocytic leukaemia. J Clin Diagn Res 2013;7:1366-71.

4. Fineberg S, Marsh E, Alfonso F, et al. Immunophenotypic evaluation of the bone marrow in non-Hodgkin's lymphoma. Hum Pathol 1993;24:636-42.

5. Horny HP, Wehrmann M, Griesser H, Tiemann M, Bültmann B, Kaiserling E. Investigation of bone marrow lymphocyte subsets in normal, reactive, and neoplastic states using paraffin-embedded biopsy specimens. Am J Clin Pathol 1993;99:142-9.

6. Bluth RF, Casey TT, McCurley TL. Differentiation of reactive from neoplastic small-cell lymphoid aggregates in paraffinembedded marrow particle preparations using L-26 (CD20) and UCHL-1 (CD45RO) monoclonal antibodies. Am J Clin Pathol 1993;99:150-6.

7. Hendry S, Salgado R, Gevaert T, et al. Assessing tumor-infiltrating lymphocytes in solid tumors: a practical review for pathologists and proposal for a standardized method from the international immuno-oncology biomarkers working group: Part 2: TILs in melanoma, gastrointestinal tract carcinomas, non-small cell lung carcinoma and mesothelioma, endometrial and ovarian carcinomas, squamous cell carcinoma of the head and neck, genitourinary carcinomas, and primary brain tumors. Adv Anat Pathol 2017;24:311-35.

8. Rao IS. Role of immunohistochemistry in lymphoma. Indian J Med Paediatr Oncol 2010;31:145-7.

9. Dewan K, Mann N, Chatterjee T. Comparing flow cytometry immunophenotypic and immunohistochemical analyses in diagnosis and prognosis of chronic lymphoproliferative disorders: experience from a Tertiary Care Center. Clin Cancer Investig J 2015;4:707-12.

10. Leers MP, Theunissen PH, Ramaekers FC, Schutte B, Nap M. Clonality assessment of lymphoproliferative disorders by multiparameter flow cytometry of paraffin-embedded tissue: an additional diagnostic tool in surgical pathology. Hum Pathol 2000;31:422-7.

11. Abdel-Ghafar AA, El Din El Telbany MA, Mahmoud HM, El-Sakhawy YN. Immunophenotyping of chronic B-cell neoplasms: flow cytometry versus immunohistochemistry. Hematol Rep 2012;4:e3. 